\title{
TRAZAS DE UNA CULTURA INSTITUCIONAL POLICIAL A TRAVÉS DE LA HISTORIA DE LA SOCIEDAD DE SOCORROS MUTUOS DE LA POLICÍA DE LA PROVINCIA DE BUENOS AIRES EN EL SIGLO XX ${ }^{1}$
}

\author{
Traces of a Police Institutional Culture through the \\ History of the Sociedad de Socorros Mutuos of the \\ Buenos Aires Province Police during the XX Century
}

\author{
Osvaldo Barreneche*
}

\begin{abstract}
RESUMEN
Este artículo se centra en la historia de la Sociedad de Socorros Mutuos de la Policía de la provincia de Buenos Aires, en especial, durante la primera mitad del siglo XX, señalando que fue en el temprano marco de dicha organización mutualista, siempre vinculada a las jefaturas de policía, donde se gestaron algunos de los fundamentos y prácticas que ayudaron a cimentar un obrar institucional de larga perdurabilidad en el tiempo. El vínculo de semi-autonomía generado entre la Sociedad, la conducción policial y el estado bonaerense, sirvió también de modelo de otras asociaciones integradas por policías y que tendrían su nacimiento y creciente influencia durante la segunda mitad del siglo XX. Precisamente a partir de mediados de dicho siglo comenzó el declinar de la Sociedad, como entidad representativa de los policías, por lo que al final del trabajo se formulan algunas hipótesis de las razones por las cuales esto puede haber ocurrido y el legado de la Sociedad en términos de la consolidación de una cultura institucional policial.
\end{abstract}

1 Una versión preliminar de este trabajo fue presentada en el IV Simposio internacional: Delitos, Policías y Justicias en América Latina. Instituto de Historia, Universidade Federal do Rio de Janeiro (IH/UFRJ) Rio de Janeiro, 2-4 março 2016.

* CONICET - IdHICS-UNLP. E-mail para contato: osvaldobarreneche@gmail.com 
Palabras-clave: Policia; socorros mútuos; Provincia de Buenos Aires; mutualismo.

\begin{abstract}
This article deals with the history of the Mutual Aid, Friendly Society of the Buenos Aires Province Police, especially during the first half of the XX Century. The piece argues that it was in the early context of this mutual aid association, always tied to the mentioned Police Agency, where some ideas of how to conduct police business took place. The bond among the Society, the Police Agency, and the Provincial State Government served as influential model for other police associations which were born later on during the second part of the same Century. Precisely from the middle of the Century on the Society began to slowly fade away as a highly representative police association. However, the Society's symbolic influence never disappeared and in this paper we would formulate some hypothesis about that endurance as well as its connections with the consolidation of a police institutional culture.
\end{abstract}

Keywords: Police; mutual aid friendly societies; Buenos Aires province; mutuality.

En los análisis contemporáneos de las ciencias sociales acerca de las policías latinoamericanas, se suele indicar que, desde comienzos del siglo XX y a lo largo del mismo, se fue conformando una cultura institucional que ha tenido un impacto importante en su historia y en sus relaciones con otras agencias estatales y con la sociedad civil. ${ }^{2}$ La policía de la provincia de Buenos Aires, en

2 Sobre este tema, ver, por ejemplo: BAILEY, John and DAMMERT, Lucía, editors (2006). Public Security and Police Reform in the Americas. Pittsburgh: University of Pittsburgh Press; DAMMERT, Lucía (2007). Perspectivas y dilemas de la seguridad ciudadana en América Latina. Quito, Ecuador: FLACSO Publicaciones; FRÜHLING, Hugo and TULCHIN, Joseph S. with GOLDING, Heather A., editors (2003). Crime and Violence in Latin America. Citizen Security, Democracy, and the State. Baltimore: Johns Hopkins University Press; GALVANI, Mariana and others (2011). A la inseguridad la hacemos entre todos. Prácticas académicas, mediáticas y policiales. Buenos Aires: Hekht Books; KAMINSKY, Gregorio and GALEANO, Diego, editors (2011). Mirada (de) Uniforme. Historia y critica de la razón policial. Buenos Aires, Teseo editores; SABET, Daniel M. (2012). Police Reform in Mexico: Informal Politics and the Challenge of Institucional Change. Stanford: Stanford University Press; SAPORI, Luís Flávio (2007). Securança pública no Brasil: desafios e perspectivas. Río de Janeiro: Editora FGV; SIRIMARCO, Mariana, comp. (2010). Estudiar la policía. La mirada de las ciencias sociales sobre la institución policial. Buenos Aires, Teseo editores; SOZZO, Máximo (2008). Inseguridad, prevención y policía. Quito: FLACSO Ecuador; TULCHIN, Joseph and RUTHENBURG, Meg, 
Argentina, no escapa a tal afirmación. Así, por ejemplo, luego de la reforma policial bonaerense ocurrida a finales de la década de 1990 se identificaron algunas de las causas por las cuales dicho proceso de transformación no pudo cumplir con todos los objetivos que se había trazado. Uno de los principales motivos señalados entonces fue la existencia y supervivencia de dicha cultura institucional, refractaria a los cambios propuestos. ${ }^{3}$ La cultura institucional policial es, entonces, frecuentemente mencionada, pero no se conocen verdaderamente sus características y su recorrido histórico. Justamente desde la historia cabe preguntarse, en principio, si dicha cultura verdaderamente existió y, de ser así, cuál fue su origen y desarrollo.

Sin duda los documentos históricos producidos por la misma policía, especialmente en los niveles superiores, remarcan una serie de ideas y de notas prácticas que permiten trazar un recorrido que puede haber dado lugar a una particular manera de gestionar y llevar a cabo las tareas policiales a lo largo del tiempo. Aun así, queda por ver, en el terreno operativo concreto, si tales pautas, como indicadoras de la cultura institucional, verdaderamente eran encarnadas y aplicadas por todos los integrantes de la agencia de seguridad bajo estudio. Por otro lado, la emergencia de tal cultura institucional no puede haberse moldeado, exclusivamente, en el marco de la propia institución. La influencia e interacción con otras agencias estatales y con diversos grupos y actores de la sociedad han sido tan influyentes como las propias ideas y prácticas policiales. Con esta importante observación a la vista, es posible aproximarse a dichos documentos policiales para explorar históricamente este tema.

Este artículo se centra en la historia de la Sociedad de Socorros Mutuos de la Policía de la provincia de Buenos Aires, en especial durante la primera mitad del siglo $\mathrm{XX}$, señalando que fue en el temprano marco de dicha organización mutualista, siempre

editors (2006). Toward a Society under Law: Citizens and Their Police in Latin America. Baltimore: Johns Hopkins University Press; UILDRIKS, Niels A. (2009). Policing Insecurity: Police Reform, Security, and Human Rights in Latin America. Lanham: Lexington Books; UNGAR, Mark (2011). Policing Democracy: Overcoming Obstacles to Citizen Security in Latin America. Baltimore: Johns Hopkins University Press.

3 Cf. SAIN, Marcelo. El Leviatán azul. Policia y politica en la Argentina. Buenos Aires, Siglo Veintiuno Editores, 2008. 
vinculada a las jefaturas de policía, donde se gestaron algunos de los fundamentos y representaciones simbólicas que ayudaron a cimentar un obrar institucional de larga perdurabilidad en el tiempo. El vínculo de semi-autonomía generado entre la Sociedad, la conducción policial y el estado bonaerense, sirvió también de modelo de otras asociaciones integradas por policías y que tendrían su nacimiento y creciente influencia durante la segunda mitad del siglo XX. Precisamente a partir de mediados de dicho siglo comenzó el declinar de la Sociedad, como entidad representativa de los policías, por lo que al final del trabajo se formulan algunas hipótesis de las razones por las cuales esto puede haber ocurrido y el legado de la Sociedad en términos de la consolidación de una cultura institucional policial.

\section{Comienzos dificiles}

La Sociedad de Socorros Mutuos de Policía nació en un contexto muy preciso durante los últimos años del siglo XIX. En una reconstrucción histórica realizada con motivo del cincuenta aniversario de su creación se mencionan algunas circunstancias que llevaron a la fundación de la misma. En primer lugar, el impulso del jefe de policía Narciso P. Lozano, quien en 1894 apoyó una iniciativa de Juan Vucetich para crear una "biblioteca de propiedad de la Policía" que habría de funcionar en la Oficina de Estadística que este último dirigía. ${ }^{4}$ La biblioteca fue un paso intermedio en la aparición de la Sociedad, pues eso "dio margen a que naciera la idea de unirse el personal de Policía y formar una entidad de Ayuda mutua, para estar en condiciones de hacer frente a los gastos que pudieran presentarse por motivo de enfermedades y fallecimientos." De hecho, en el mismo despacho del jefe Lozano se llevó a cabo, el 29 de septiembre de 1894, "una reunión de funcionarios de la Repartición, 
quienes después de un agitado debate dejan constituida [..] la Sociedad de Socorros Mutuos y Biblioteca de Policía." 5

$\mathrm{Si}$ bien no contamos con registros del aludido "agitado debate", seguramente estas palabras reflejaron la preocupación de los jefes policiales de entonces por encontrar soluciones a las exiguas cualidades profesionales del personal subordinado, agravadas por la precariedad de sus condiciones materiales de vida. El mismo documento refiere al contexto de la crisis y revoluciones de $1890 \mathrm{y}$ 1893, "épocas turbulentas [que] trajeron aparejadas una serie de consecuencias graves [...] creando problemas que estaban muy lejos de solucionarse con los exiguos sueldos que por ese entonces devengaban los empleados de la Administración, y en forma especial el personal de Policía". 6

Los estatutos de la nueva entidad sufrieron varias modificaciones y ajustes durante las décadas iniciales de existencia. Visto desde épocas posteriores, cuando se consideraba que era el estado el encargado de proveer los servicios sociales básicos a sus empleados, el ideario mutualista basado en el "principio de compañerismo", plasmado en aquellas primeras obligaciones estatutarias, habría provocado "ingentes pérdidas a la Sociedad, originando sus primeros desastres financieros". En realidad, los registros existentes ponen en evidencia que la masa societaria era exigua respecto a las necesidades a cubrir. Durante sus primeros años de vida la Sociedad no superaba los 100 afiliados. Luego ese número fue en aumento, pero con una gran disparidad, en cuanto que resultaba muy difícil mantener el cobro de las cuotas de manera regular y al día. ${ }^{7}$

5 Reseña Histórica de la Sociedad de Socorros Mutuos de Policía de la provincia de Buenos Aires escrita por su presidente Don Rómulo Méndez Caldeira en ocasión del 50 aniversario de su fundación. 1894 - 29 de septiembre - 1944, publicado en el Libro de Oro de la entidad en 2014 , p. 40

6 Ibidem p. 35. Sobre la inestabilidad laboral del personal y las dificultades que representaba para los jefes el mantenimiento de un plantel estable de policías puede verse GAYOL, Sandra, "Entre lo deseable y lo posible. Perfil de la Policía de Buenos Aires en la segunda mitad del siglo XIX”, En: Estudios Sociales, Año VI, número 10, Santa Fe, primer semestre de 1996, pp. 123 138.

7 Reseña Histórica, página 46 a 53. Sobre algunas formas asociativas de ayuda mutua y otras de asistencia social anteriores a la emergencia del Estado de Bienestar, puede verse el libro de MORENO, José Luis (comp.). La politica social antes de la política social (Caridad, beneficencia y 
Algunas propuestas de la Sociedad, incluidas en sus estatutos, no alcanzaron a concretarse, o bien tuvieron corta vida, o no siempre pudieron sostenerse sobre la base de la cuota societaria. Por ejemplo, el Banco Policial ideado en 1915, nunca se creó. La Caja de Ahorros y Préstamos o la Caja de Descuentos siguieron el derrotero de las finanzas societarias, originando diversas reacciones de expansión y reducción en las sucesivas Asambleas. El proyecto de apertura de una Farmacia Social y de Consultorios Médicos no pasó de la instancia de estudio de factibilidad que se la había encomendado a una comisión especial. Y la Revista de Policía creada por la Sociedad en 1910, de aparición mensual, tuvo solo dos años de duración en esta primera etapa, pues "sus entradas no compensan las salidas". 8

Transcurridas las primeras tres décadas de su existencia, la Sociedad de Socorros Mutuos no había logrado consolidarse institucionalmente. El propósito de asistir a los policías de entonces aun no podía cumplirse y las Asambleas realizadas daban cuenta del déficit crónico de la entidad. Frente a dicho panorama, los policías no se veían particularmente atraídos a asociarse y participar. Los beneficios obtenidos eran escasos y constantemente suspendidos por falta de fondos, como en la Asamblea del 11 de junio de 1920, cuando se resolvió cortar "la entrega de medicamentos y asistencia a los enfermos venéreo-sifilíticos". Y no faltaban tampoco los problemas por desviación o apropiación indebida de dineros de la Sociedad, lo cual minaba su reputación. Así, en la Asamblea del 3 de diciembre de 1932 se informó del suicidio del tesorero de la Junta Ejecutiva al tiempo que se descubría un faltante de ocho mil pesos del fondo social de la entidad. ${ }^{9}$

Tal panorama hizo a la Sociedad muy dependiente, en términos económicos, de la jefatura de policía y del gobierno provincial. La suscripción de socios, rifas, kermeses y otras

politica social en Buenos Aires, siglos XVII a XX). Buenos Aires, Editoriales Trama y Prometeo Libros, 2000.

8 Según informes de la Sociedad, se editaron 36 números de la primera serie de la Revista, entre julio de 1900 y diciembre de 1902. La propuesta original era de frecuencia quincenal, pero esto no pudo sostenerse durante esos dos años. 1932.

9 Sociedad de Socorros Mutuos de Policía. Documentos de archivo. Asambleas, 1920 y 
actividades no alcanzaban para cubrir los objetivos estatutarios de la entidad. Al mismo tiempo, muchos requerimientos y necesidades no podían ser satisfechos, lo cual hacía apoyar más las finanzas en las donaciones y subsidios permanentes para la asistencia social de los afiliados. Las sucesivas conducciones policiales, comenzando por aquella que propició el nacimiento de la Sociedad, eran conscientes del rol que cumplía una entidad "hermana" que podía dar respuestas rápidas a problemas concretos de los policías, sin tener que recurrir a la burocracia. Sin embargo, no todos los jefes de policía se comprometían de igual manera. Por ello la Sociedad iba a recordar y reconocer especialmente a Luis María Doyhenard, quien fuera jefe de policía y dos veces presidente de la Sociedad (1902-1906 y 1916), como uno de los que supo sostener el mutualismo policial en momentos difíciles. ${ }^{10}$

Encontramos además un gran contraste entre la permanencia en el cargo de los presidentes de la Sociedad hasta los años treinta, y de allí en adelante. Dieciséis fueron los presidentes desde 1894 hasta 1933, un promedio de poco más de dos años cada uno. Observando las fechas de cada presidencia, se nota que la sucesión de estos 16 presidentes no fue siempre producto de las finalizaciones de sus mandatos sino más bien respondieron a los cambios políticos y de conducción de la jefatura de policía. Allí también puede verse el vínculo fuerte que existía entre las autoridades de la Sociedad y la conducción policial de turno. ${ }^{11}$

\section{En búsqueda de la estabilidad}

A comienzos de los años treinta cambió la frecuencia de recambio de las autoridades de la Sociedad. A partir de 1933 hasta la

10 Además de ayudas concretas, Doyhenard también impulsó la iniciativa del panteón social, por lo que, a poco de inaugurarse el mismo, sus restos fueron trasladados a la nueva bóveda. Sociedad de Socorros Mutuos de Policía. Lista de presidentes de la entidad desde su fundación en 1894 hasta la actualidad.

11 Los continuos cambios de Estatuto iban adecuando la duración de los mandatos, inicialmente previstos con una duración de dos años, con renovaciones. 
actualidad, solamente 7 personas han ejercido la presidencia, dos de las cuales lo hicieron "a cargo" por menos de un año, debido al fallecimiento del presidente. De los cinco restantes, sobresalen las sucesivas reelecciones de Rómulo Méndez Caldeira (presidente entre 1933 y 1948) y Carlos M. Spinosa (presidente entre 1948 y 1997). ${ }^{12}$ La gestión de Méndez Caldeira y sus vínculos con la dirigencia política conservadora le permitieron dar continuidad a su mandato más allá de los sucesivos cambios políticos de la década. Una de las primeras medidas del nuevo presidente fue gestionar y obtener una subvención mensual de parte del gobierno, la cual fue incluida en el presupuesto anual de la provincia. Esta y otras ayudas y donaciones fueron estabilizando las finanzas de la Sociedad. Además, la cantidad de socios fue en aumento, pasando de 2012 en 1934 a 3300 en $1938 .{ }^{13}$ Si tomamos en cuenta que la cantidad total de policías en 1933 era de 10.221 , ascendiendo a 14.066 para 1943, podemos pensar que la cobertura de la Sociedad solo alcanzaba a, aproximadamente, un $20 \%$ del personal policial. ${ }^{14}$

Los gobiernos conservadores aun no otorgaban beneficios sociales generales al personal de la institución. La paga local, por comisaría, y la posibilidad de administrar las altas y bajas de cada dependencia, hacía que su personal fuera más dependiente de las autoridades policiales y políticas locales y municipales, de las cuales también obtenían ayuda para paliar sus necesidades personales y familiares. Si bien no contamos con la distribución regional de los asociados, los informes y documentos de la Sociedad indican que su mayor radio de acción era La Plata y alrededores, con alguna presencia en otras ciudades bonaerenses. Eso hacía que los beneficios otorgados no llegasen a todos los policías bonaerenses como bien señalan las cifras ya marcadas. Por su parte, los sucesivos

12 Sociedad de Socorros Mutuos de Policía. Nómina de sus Presidentes desde la fundación (1894) hasta la actualidad (2014).

13 Con altibajos, la cantidad de socios fue incrementándose desde principios de siglo XX. Para la segunda mitad de la década de 1920 el promedio de socios era de 1500, por lo que los mismos se duplicaron en la década siguiente. Reseña Histórica de la Sociedad de Socorros Mutuos de Policia de la provincia de Buenos Aires, Op.Cit. p. 62-63.

14 Evolución histórica de la fuerza efectiva de la Policía de la Provincia de Buenos Aires, 1933-2005". En: Departamento Estadística. Dirección Provincial de Personal de la Provincia de Buenos Aires. 
gobernadores, y especialmente Manuel Antonio Fresco (1936-1940), intentaron modificar esta situación, procurando una mayor subordinación de la policía a la conducción provincial. ${ }^{15}$ Pero mientras esto no ocurría, tampoco quisieron ampliar beneficios aunque si dieron un fuerte respaldo a los planes y objetivos de la Sociedad.

Por entonces, la creación de dos espacios físicos administrados por la entidad, vinculados a la vida y la muerte, iban a servir de pilares para afianzar su influencia.

\section{Un vínculo más fuerte que la muerte}

La adquisición de una sede social permanente en la ciudad de La Plata y la construcción del "panteón social" en el cementerio de dicha ciudad resultaban ser dos metas buscadas prácticamente desde los orígenes de la Sociedad. Los asientos de la entidad funcionaron en diversas locaciones de la ciudad hasta que, por fin, a finales de 1943 pudo adquirirse el inmueble de la calle 59 entre 6 y 7 que ha servido de sede desde entonces. Una donación de títulos de la provincia de Buenos Aires por quinientos mil pesos dejados en el testamento de Luis A. Repetto, fallecido en septiembre de 1940, dotó a la Sociedad con una inesperada suma de cuyos intereses se valió para reunir parte de los fondos necesarios en la compra de la nueva sede. ${ }^{16}$ Otra parte del dinero fue cubierta por un préstamo solicitado al Banco de la

15 Sobre este tema puede verse mi trabajo sobre la reforma policial iniciada durante la gobernación de Fresco: "De brava a dura. La policía de la provincia de Buenos Aires durante la primera mitad del siglo XX". En: Cuadernos de Antropología Social. Sección de Antropología Social, Instituto de Ciencias Antropológicas. Facultad de Filosofia y Letras, Universidad de Buenos Aires, Número 32, Diciembre 2010, pp. 31-56.

16 En la síntesis histórica de la Sociedad se transcribe el testamento del Sr. Repetto, quien además de dejar algunos inmuebles a favor de sus hermanas y sobrinas políticas, detalla numerosas propiedades, sumas de dinero y títulos públicos que dejase a hospitales, instituciones públicas y privadas, particulares, y sociedades como la de Socorros Mutuos de Policía, sin que pudiese conocerse, en este último caso, los motivos que llevaron a tal donación. Cf. Pag. 69-70. La Sociedad reconoció el aporte de Luis A. Repetto dando su nombre a la Biblioteca de la entidad. 
Provincia de Buenos Aires, con el aval del gobierno provincial. De este modo, se estableció un punto fijo de referencia, en la ciudad de La Plata, donde funcionaría permanentemente la Sociedad y a donde se trasladaron los consultorios médicos, la biblioteca y otros servicios que prestaba. Desde entonces, el sitio fue frecuentado por los asociados y sus familiares, tornándose en un lugar reconocible para los policías, especialmente aquellos residentes en la capital provincial y zonas de influencia.

Mayor impacto simbólico tuvo la construcción e inauguración del panteón policial bonaerense en el cementerio municipal de La Plata en $1940 .{ }^{17}$ En las resoluciones de las juntas ejecutivas de la Sociedad encontramos, desde 1902, antecedentes sobre la búsqueda de fondos para dicho emprendimiento. Existen, también, planos y bocetos de los proyectos presentados. En los dos más desarrollados, de 1904 y 1923 respectivamente, notamos un estilo muy ornamentado y cargado que remataba en cúpula. El diseño era similar al panteón militar, de principios de siglo XX, y al de la entonces Policía de la Capital, inaugurado en 1922, ambos situados en el cementerio de la Chacarita. En su estudio sobre estos últimos, Diego Galeano señala que esta coincidencia no era casual, pues "los policías reclamaban un lugar en el culto necrológico a los héroes de la patria." 18

En base a esos proyectos, en 1913 se alcanzó a colocar la piedra fundamental del "Panteón Guardianes del Orden Público" en un sitio del cementerio de La Plata cedido por el Concejo Deliberante. Sin embargo, este nunca se construyó y la cesión municipal terminó caducando. El motivo principal de este fallido tuvo que ver con la ya señalada situación financiera de la Sociedad en aquellas décadas. Por un tiempo se lograba acumular una suma de dinero, por donaciones, rifas, etc. destinada especialmente a la construcción del panteón. Sin

17 Sobre los diversos significados de la muerte y su culto, en el contexto latinoamericano, puede verse el libro editado por JOHNSON, Lyman L Death, Dismemberment, and Memory. Body Politics in Latin America. Albuquerque, University of New Mexico Press, 2004.

18 Cf. GALEANO, Diego, "Caídos en el cumplimiento del deber". Notas sobre la construcción del heroísmo policial”, En: GALEANO, Diego y KAMINSKY, Gregorio (coordinadores), Mirada (de) Uniforme. Historia y crítica a la razón policial. Buenos Aires, Editorial Teseo y UNLa, 2011, p. 207. 
embargo, tarde o temprano, la junta ejecutiva de turno debía echar mano a ese dinero para cubrir el déficit crónico de la entidad.

El culto a los muertos, los entierros, las tumbas y monumentos dentro y fuera de los cementerios, atravesaban entonces un proceso de transformación que señalaba claramente el uso político de todo ello, al tiempo que revelaba nuevas estéticas y sensibilidades sociales en la Argentina de principios del siglo XX. Así, los diseños aludidos respondían a un nuevo ritual celebratorio que era acompañado por la monumentalidad de las tumbas. Sandra Gayol, Gabriel Kessler y otros han estudiado este tema, señalando que el estado no ha sido ajeno a la "función unificadora de los ritos mortuorios." 19 Ellos también muestran los cambios producidos a lo largo del siglo XX hasta la actualidad. En ese sentido, el caso del panteón policial bonaerense sigue los parámetros descriptos por estos autores. En efecto, cuando recobró fuerza este proyecto, hacia finales de los años treinta, el estado provincial y los personajes destacados de la política bonaerense dieron un fuerte impulso para su concreción. Si bien la Sociedad había vuelto a reunir dinero para este propósito, fueron las donaciones del Gobernador Manuel Fresco y del Intendente de Avellaneda, Alberto Barceló, las que en 1938 permitieron finalmente el inicio de las obras. La Municipalidad de La Plata volvió a ceder un terreno en la necrópolis local, colocándose la nueva piedra fundamental en mayo de 1939 e inaugurándose en octubre de $1940 .^{20}$

El panteón policial bonaerense resulta estéticamente muy diferente a aquellos de las otras fuerzas militares y policiales inaugurados en las décadas previas. Es de un estilo simple, sin ornamentos, con un techo originalmente plano, donde solo sobresale la representación de una llama votiva. Con la ampliación proyectada desde la década de 1960 y finalmente culminada en 1985, se le construyó un segundo piso coronado por un tinglado de chapa como techo, por lo que la llama quedó alojada dentro de la pared frontal. Las formas sobrias del panteón policial están acordes a la época de su construcción, en la cual la política de masas y los "funerales

19 GAYOL, Sandra y KESSLER, Gabriel (Editores), Muerte, politica y sociedad en la Argentina. Buenos Aires, Editorial Edhasa, 2015; Introducción, p. 10.

20 Reseña Histórica de la Sociedad de Socorros Mutuos de Policía de la provincia de Buenos Aires, Op.Cit. p. 117 
republicanos" marcaron distancia respecto a la exaltación individual del muerto ilustre o heroico y su visibilidad en el sitio elegido para su eterno reposo, que había sido la característica saliente a comienzos del siglo. ${ }^{2}$

Inmediatamente de inaugurado, el panteón policial tuvo su reglamento (aun vigente) por el cual cada nicho conservaría un ataúd por 20 años, para luego reducir los restos y conservarlos en el sector del panteón destinado a tal fin. Tal como indicase el presidente de la Sociedad, Rómulo Méndez Caldeira, en su discurso inaugural, rodeado de autoridades políticas y policiales, "ni el tiempo ni cualquier circunstancia, hará que un empleado de policía de la Provincia que forme parte de la Entidad o caído en medio de la lucha diaria contra el delito, o cualquier allegado a los mismos y que perteneciera a nuestra Institución, será olvidado; sus restos serán conservados eternamente dentro del Panteón". 22 En efecto, el acceso al sitio policial del descanso póstumo se destinó a los asociados y familiares pero también incluyó a los "caídos en el cumplimiento del deber." Sin embargo, el reglamento estableció que todos los nichos fuesen iguales, indicándose solamente el nombre del difunto y la fecha de fallecimiento, no las causas o circunstancias de la muerte.

En el panteón policial puede verse la uniformidad de los nichos situados las paredes laterales de la capilla localizada en la planta baja central. ${ }^{23}$ Hacia el fondo, en una ubicación ordinaria, se encuentran depositados los restos de Juan Vucetich, fallecido y sepultado en Dolores en 1925, y trasladado al panteón policial en 1941. El ilustre policía es el único cuyo cadáver no fue reducido luego de los 20 años. Esta es la excepción establecida por la Sociedad para el más famoso de sus miembros y quien fuera, además, su primer presidente. Sin embargo, su féretro está allí como uno más, rodeado de policías solo conocidos por sus allegados más cercanos. Como señalaba en su discurso el presidente Mendez Caldeira, "se buscó

21 Al respecto, véase GAYOL, Sandra, "Ritual fúnebre y movilización política en la Argentina de los años treinta", En: PolHis, Año 6, Número 12, Segundo semestre de 2013, pp. 225 243.

22 Rómulo MENDEZ CALDEIRA, discurso pronunciado con motivo de la inauguración del Panteón Policial. 18 de octubre de 1940. Sociedad de Socorros Mutuos de Policía.

23 En los otros pisos del panteón existe una disposición igualmente uniforme, solamente que en el lugar que ocupa la capilla de planta baja, hay otra hilera de nichos. 
uniformidad en todo," infiriéndose de sus palabras que las diferencias jerárquicas y de otro tipo que tanto distinguían (y aun distinguen) a la policía bonaerense, caducaban en la puerta del panteón. Allí los policías muertos, naturalmente o "en cumplimiento del deber", eran todos iguales.

El panteón tuvo un impacto importante como lugar simbólico, y a la vez concreto, para la policía bonaerense de entonces, aun cuando este impacto menguaría en el tiempo. Fue un lugar de encuentro "igualador" para todos sus miembros. Un sitio de homenaje, de memoria y también de culto, pues hasta hoy se celebran allí periódicas ceremonias religiosas del rito católico. En esa morada colectiva, los cuerpos de un jefe policial y del más raso de los agentes yacen uno al lado del otro, o tal vez contiguos a los restos de un familiar de otro policía. Tal vez es por esto mismo que la estética de igualdad mutualista iba a contrastar con una crecientemente jerarquizada institución, lo cual comenzó a evidenciarse, por ejemplo, en un ceremonial funerario diverso de acuerdo a los rangos policiales.

\section{Un medio para varios fines}

En el contexto de creciente estabilidad institucional y metas alcanzadas de comienzos de la década de 1940, especialmente con la ayuda del estado provincial, la Junta Ejecutiva presidida por Méndez Caldeira pudo reeditar la Revista que, como se indicó, había tenido una corta existencia en los inicios de Sociedad, entre 1910 y 1912. La nueva era de la publicación nació gracias a un fuerte respaldo de la Jefatura de Policía. Así lo agradeció la dirección en su primer editorial, reconociendo el apoyo recibido por el entonces jefe de policía, Coronel (r) Enrique Rottjer. ${ }^{24}$ De hecho, la publicación

24 El jefe de policía Rottjer concretó varias de las propuestas que se venían considerando desde antes en el marco de la profesionalización de la policía. Así, fue el creador de la Escuela de Oficiales de Policía, inaugurada en agosto de 1941. Ver "Escuela de Policía; su inauguración". Orden del Día Número 15.369. Agosto 12 de 1941. Partidario de las ideas políticas 
apareció como Revista de Policía de la Provincia de Buenos Aires, "publicación oficial mensual auspiciada por la Jefatura de Policía de la provincia". Solo el sello de la Sociedad, incorporado en la portada, daba a entender su origen institucional. Por lo demás, la Revista parecía editada por la policía misma. De hecho, casi lo era pues la dirección contaba con un representante de la Sociedad y otro puesto por la jefatura de policía. Entre sus objetivos se proponía incluir artículos de especialistas en criminología, estadística criminal, identificación personal, técnica jurídica procesal, la psiquiatría, la sociología y la ética profesional. Sin embargo, mucho de su contenido refería más a destacar los "logros" de la policía, las acciones y discursos del jefe Rottjer, los aportes de la Sociedad de Socorros Mutuos al bienestar policial y las efemérides institucionales y patrias. $^{25}$

El "apoyo" de la jefatura policial a la Revista puede verse también en su creciente publicidad. Además de los avisos del Banco de la Nación Argentina y del de la Provincia de Buenos Aires, la publicación contó ya desde su número 2 con auspicios de entidades del interior bonaerense, aun cuando la circulación todavía no había alcanzado una gran amplitud territorial. Esta pauta venía de la mano de una campaña de suscripciones realizada por las autoridades policiales. Así, para el número 4, ya existía una gran diversidad publicitaria de empresas, comercios, fábricas, compañías de servicios, hoteles, todos ellos de varias ciudades bonaerenses. ${ }^{26}$ Desde su segunda aparición la Revista conservó su nombre pero con la aclaración, en la portada, de que era editada por la Sociedad. En los números siguientes se agregó la aclaración de que se trataba de una

del General Uriburu, a quien acompañó en el golpe militar que derrocó a Hipólito Irigoyen el 6 de Septiembre de 1930, el Coronel (R) Enrique Rottjer fue dos veces interventor del gobierno nacional de la Concordancia en la provincia de Buenos Aires y dos veces jefes de policía, todo ello durante el año 1941 en que se creó la escuela de policía. Del 15 de Febrero al 2 de septiembre de 1941 fue jefe de policía; del 2 al 13 de septiembre fue interventor federal en la gobernación; del 13 de septiembre al 21 de octubre fue nuevamente jefe de policía; y del 21 de octubre hasta el 7 de enero de 1942 fue nuevamente interventor federal hasta la asunción del nuevo gobernador Rodolfo Moreno. Ver BEJAR, María Dolores, El régimen fraudulento. La política en la provincia de Buenos Aires, 1930 1943. Buenos Aires: Siglo XXI Editores, 2005, pp. 178-186.

25 Revista de Policía de la Provincia de Buenos Aires. Año I, número 1, Mayo de 1941.

26 En el número 2 de la Revista, por ejemplo, hay una página entera de avisos correspondientes a la ciudad de Pergamino. 
"publicación no oficial" aunque en la página 1, junto a los créditos y el índice, se indicaba que estaba "auspiciada por la Jefatura de Policía". Finalmente, en su número 42 del año 1944, pasó a llamarse directamente "Revista de la Sociedad de Socorros Mutuos de Policía". En el editorial de ese número se aclaró que el cambio de nombre era para adecuarse al Decreto 24.381 del gobierno nacional militar, que prohibía el uso de "policía" o "policial" a revistas o publicaciones particulares. ${ }^{27}$

Aun cuando se procuraron estas distinciones, el vínculo bonaerense entre Sociedad de Socorros Mutuos y Jefatura de Policía siguió siendo cercano. En el número 44 de 1944 se informa, por ejemplo, del cambio del director de la publicación. Para ello, se reproduce la nota enviada por la Sociedad al jefe de policía solicitando designe un reemplazante, y la respuesta del jefe policial nombrando al Comisario de la División Judicial Luis María Althabe para esa función. ${ }^{28}$ De todos modos, la diferencia entre la institución policial y la Sociedad resultaba también apropiada para el tratamiento de ciertos temas. Un editorial de 1945, por ejemplo, reclamaba una compensación por gastos de traslado para todo el personal policial que debía cambiar de destino, opinando sobre la creación de una oficina en el Ministerio de Hacienda provincial destinada a tal fin. Una opinión abierta sobre el tema solo podía permitirse en ese ámbito, dado que los policías no estaban autorizados a peticionar beneficios y compensaciones. ${ }^{29}$

La llegada del peronismo al poder no alteró inicialmente el funcionamiento de la Sociedad y de su revista. Como tantos, el presidente de la Junta Ejecutiva, Mendez Caldeira, apoyó el ascenso

27 Cf. Revista de la Sociedad de Socorros Mutuos de Policia, año 1944, número 42, página 1. La sanción de este decreto del gobierno nacional no estaba destinada particularmente a la publicación de la Sociedad, sino a evitar lo que había ocurrido hasta entonces, especialmente en la ciudad de Buenos Aires, con revistas de policía que, bajo ese título, pero como iniciativas particulares, eran vendidas por suscripción dentro de la policía y la justicia. Reservando el nombre "Policía" para las publicaciones oficiales, el gobierno se proponía editar sus propias publicaciones institucionales, tal como ocurrió, de manera intermitente, durante los años subsiguientes hasta comienzos de la década de 1960. Cf. Decreto PEN 24.381 del 1 de septiembre de 1944.

28 Cf. Revista de la Sociedad de Socorros Mutuos de Policía, año 1944, número 44, página 16.

29 Cf. Revista de la Sociedad de Socorros Mutuos de Policía, año 1945, número 47, página 1. Esta compensación por gastos de traslado fue luego otorgada por el peronismo. 
de Perón durante los años del gobierno militar. Especialmente desde 1944 publicaron noticias sobre las actividades de Perón a favor de diversos sectores sociales. ${ }^{30}$ Poco después, en 1948, el fallecimiento de Mendez Caldeira dio paso al ascenso de Carlos M. Spinosa, quien sería el nuevo presidente de la Sociedad por los próximos 49 años, hasta su muerte acaecida en 1997. Si bien aun se conoce poco sobre la vida de Spinosa, algunas noticias marcan el favor del gobierno peronista hacia su persona, lo cual precedió su encumbramiento como presidente de la Junta Ejecutiva de la Sociedad. En febrero de 1948, pocos meses antes de la muerte de Mendez Caldeira, y ocupando ya el cargo de secretario de la Junta Ejecutiva, la Revista informó del ascenso de Spinosa, quien pasó de ser auxiliar mayor a desempeñarse como Subcomisario, segundo jefe de Suministro y Contralor de Policía. ${ }^{31}$

Fue durante esos mismos años del primer peronismo en lo que comenzó el estancamiento y eventual declinación de la Sociedad de Socorros Mutuos de Policía. El proceso de institucionalización madurado a partir de la década del treinta fue profundizado por el gobierno militar y consolidado por la reforma policial llevada a cabo durante la gobernación de Domingo Mercante desde 1946. Más allá de las diferencias y especificidades de cada etapa, la creciente complejidad de la organización policial bonaerense acompañó un proceso de centralización y verticalidad en el control institucional. La asignación de beneficios sociales a todos los policías, iniciada por el gobierno militar desde 1944 y completada durante el peronismo, hizo que muchos de los servicios ofrecidos por la Sociedad pasasen a ser complementarios y secundarios. Si el nivel de asociados nunca representó más de una cuarta parte de toda la fuerza policial aun en su momento de mayor crecimiento durante los años treinta, la Sociedad iba a ver declinar ese porcentaje desde entonces por todo el resto de su historia hasta la actualidad. número 39.

30 Por ejemplo, en Revista de la Sociedad de Socorros Mutuos de Policía, año 1944,

31 Revista de la Sociedad de Socorros Mutuos de Policía, año 1948, número 78. Mendez Caldeira falleció el 25 de junio de 1948 según informa el número 80 de la Revista (p. 6). Poco después, Spinosa fue elegido presidente. 
En aquel momento de mediados de siglo también se verificó el nacimiento de diversas asociaciones destinadas a e integradas por policías que pueden haber sido vistas como una competencia para la Sociedad (Círculo de Oficiales, de Suboficiales, de Retirados). Las mismas, como se indicó, respondieron a la creciente jerarquización de la fuerza. A diferencia de la Sociedad, que englobaba a todos los policías por igual, estas nuevas agrupaciones tenían un derecho de admisión más acotado. La más destacada y perdurable fue el "Circulo Policial", fundado en 1945. La invitación a hacerse socio mencionaba fundamentos tales como "promover el conocimiento mutuo, estrechar vínculos amistosos, fomentando además las reuniones sociales y culturales de sus familias." Y si bien se abría la propuesta invitando al "Señor empleado o jubilado de policía: contribuya a esta obra de acercamiento espiritual. Hágase socio," lo cierto es que este espacio quedaría solamente reservado para el nuevo escalafón de Oficiales. ${ }^{32}$

La Sociedad de Socorros Mutuos de Policía no tenía entre sus objetivos de entonces el promover encuentros recreativos y espacios de sociabilidad. Su propuesta seguía marcada por la asistencia social de sus afiliados, que cubrían tanto la vida como la muerte de los mismos según se indicó. Es por ello que desde las páginas de la Revista se promovió el naciente Circulo Policial, sin que se interpretase entonces como una competencia. En tal caso, la declinación de la Sociedad estuvo acompañada por el retiro del apoyo institucional y financiero que el estado provincial y la jefatura de policía le habían brindado hasta entonces. Esto fue acompañado por una merma de afiliados cuya asistencia social, en caso de necesidad, provenía directamente de la institución policial. ${ }^{33}$ Claro está que este proceso no tomó a la Sociedad aisladamente, sino que se inscribió en el contexto de la lenta retracción del cooperativismo durante la segunda mitad del siglo $\mathrm{XX}^{34}$

32 Revista de la Sociedad de Socorros Mutuos de Policia, año 1945, número 47, página 12. Al nacimiento del Circulo Policial le seguirían el Circulo de Suboficiales, el de Retirados, etc.

33 La creación de los "Servicios Sociales" de Policía, hacia finales de los años cincuenta completó ese proceso.

34 Sobre este tema, su contexto y su bibliografía, aun cuando luego se focaliza en el ámbito rural, puede verse el capítulo de MATEO, Graciela, "La experiencia cooperativa en el agro bonaerense", en: BARRENECHE, Osvaldo (director del tomo). Historia de la provincia de Buenos 
Sin embargo, la Sociedad de Socorros Mutuos de Policía se mantuvo a pesar de estas dificultades. En el editorial de la Revista de 1994, año del centenario de la Sociedad, al destacar los momentos trascendentes del siglo transcurrido, su vicepresidente Lapalma mencionó varios hitos cruciales. Entre ellos, el de finales del primer peronismo, cuando "la Jefatura de Policía absorbió, no obstante que fue decisión de sus socios por asamblea, a dos instituciones integradas por personal de la Policía de la provincia, cuales fueron el Circulo Policial y la Cooperativa del Personal Policial - COPOBA -; pero la Sociedad de Socorros Mutuos resistió toda presión que se ejerció para que siguiera ese camino y mantuvo su individualidad. Por ese hecho sufrió cierta persecución que no afectó su desenvolvimiento". 35

Si repasamos rápidamente todo lo ocurrido en la historia argentina y bonaerense entre los años 1948 y 1997, durante los cuales la Sociedad tuvo como presidente de su Junta Ejecutiva a Carlos M. Spinoza, podemos conjeturar sobre la habilidad política del citado para sortear todos los cambios, marchas y contramarchas que acontecieron durante esos cinco lustros. El momento histórico referido por la editorial de la edición centenaria bien puede haber sido uno de ellos. Sin embargo, también puede especularse con la pérdida de peso político e institucional que la Sociedad experimentó desde mediados del siglo pasado.

\section{Consideraciones finales}

Hemos visto que la apelación al mutualismo y la cooperación nunca habían despertado un gran entusiasmo entre los policías de las primeras décadas del siglo XX. Aun así, estos acompañaron los esfuerzos de la Sociedad por brindar un único espacio de asistencia a

Aires. Volumen 5: del primer peronismo a la crisis de 2001. Buenos Aires, Edhasa y Unipe, 2014, pp. 359-382.

35 Revista de la Sociedad de Socorros Mutuos de Policía, año XXXI, 4ta época, número 99, enero a diciembre de 1994, página 3. 
los vivos y de descanso póstumo a los muertos. Pero la jerarquización y verticalidad que siguió el proceso de profesionalización desde, al menos, los años treinta en adelante, maduró a mediados de la centuria en una oficialidad que más bien buscaba una clara distinción entre ellos y la "tropa" policial. Estas nuevas camadas, surgidas de la Escuela de Policía, no iban a aceptar tan fácilmente tales espacios en común. Es por eso que el Circulo Policial y otras agrupaciones concitaron una mayor adhesión desde entonces y resultaron mejores cajas de resonancia sobre lo que los oficiales y jefes policiales tenían para decir. Tal vez, por eso mismo, el interés de las sucesivas jefaturas de policía y de los gobiernos bonaerenses estuvo centrado en los nuevos espacios de socialibilidad y no en la más tradicional Sociedad de Socorros Mutuos que los había precedido.

Pero al mismo tiempo, la supervivencia y persistencia de la Sociedad le permitió retener para sí el patrimonio de algunas ideas y prácticas, especialmente en términos de representaciones simbólicas, que constituyeron aspectos comunes a toda la policía. De este modo, la versatilidad de la cultura institucional construida históricamente, le debe a la Sociedad mucho de su impronta inicial que pudo darle, al mismo tiempo, la rigidez y flexibilidad en la cual habría de forjarse y consolidarse durante el resto del siglo. Así, no como tribuna pero si como lugar emblemático, abonado por varios hechos de alto impacto como que su fundador fue el mismísimo Vucetich, la Sociedad de Socorros Mutuos de Policía representa aun hoy ese espacio a la vez universal y específicamente policial al cual siempre puede apelarse para buscar darle sentido a lo que cada quien, que cree representar a "la policía", entiende que y cómo debe ser la misma.

RECEBIDO EM: 15/05/2016 APROVADO EM: 20/06/2016 